\title{
High frequency ultrasound study of skin tumors in dermatological and aesthetic practice.
}

\section{Artur Bezugly}

Moscow Scientific-Practical Center of Dermatovenerology and Cosmetology, Moscow, Russia.

\begin{abstract}
The accurate measurement of skin tumors and the precise delimitation of its borders areimportant tools for tumor diagnosis and treatment. In this paper we summarized our practical experience in 'the examination of different skin tumors using high frequency ultrasound (HFU). High-frequency transducers $22 \mathrm{MHz}$ and $75 \mathrm{MHz}$ with resolution of 72 and $21 \mu \mathrm{m}$ were used for the examination. HFU characteristics of the most important non-melanoma skin tumors are depicted.
\end{abstract}

Keywords: high frequency ultrasound, skin tumors, diagnosis

Dermatologists and cosmetologists are one of the first specialists carrying out the diagnosis and treatment of benign and malignant skin tumors. Currently for instrumental diagnostics of tumors dermatoscopy is most often used. This methodology is universally a standard procedure. As an optical technique, dermoscopy has a high resolution, but in virtue of the laws of propagation, reflection and diffusion of light in turbid media, the depth of the dermatoscopic study does not exceed 350-500 $\mu \mathrm{m}$. The information regarding localization, depth, and internal borders of the tumor with surrounding tissues are very important for diagnosis and treatment planning. So, for the study of skin tumors, especially for nonmelanocytic tumors, additional tools are required.

One of the objective quantitative methods for the study of normal skin and skin pathologies is high-frequency ultrasound (HFU) scanning. The resolution of the method is quite high, ranging from 80 to $16 \mu \mathrm{m}$, the depth of penetration into the skin in the range of $20-100 \mathrm{MHz}$ $12-3 \mathrm{~mm}$, which is sufficient for the study of epidermis,

Received 30.09.2015 Accepted 25.10.2015

Med Ultrason

2015, Vol. 17, No 4, 541-544

Corresponding author: Artur Bezugly

16-382 Pyatnitskoe hgw,

Moscow, 125464 Russia

Phone: +7 916201 9009, Fax: +7 4957544674

E-mail: DrArturBezugly@gmail.com dermis and subcutaneous fat [1]. Conventional scanners used in general ultrasonography have a lower resolution and, in most cases, cannot be used for visualization of the fine morphological structures of the skin. One of the main advantages of HFU is the high resolution, comparable with histology [2].

Reflection of the ultrasonic waves from the boundary between the tissues with different acoustic properties is the basis for ultrasound imaging of the skin. This principle allows to differentiate tissues with only $0.1 \%$ density difference. Usually mechanical applicators containing one piezoelectic element (fig 1) are used for the skin study. In this mode a vertical section of the skin is obtained.

When moving over the skin, the piezoelectic element operates in pulse-echo with a high frequency and the amplitude of the reflected ultrasound beam is converted into a color pixel. The color of the pixel depends on the amplitude of the reflected signal. One pulse-echo brings one amplitude curve or A-scan (fig 2). If the A-scan curve is analyzed from left to right, each part of this curve corresponds to a tissue-specific point situated on the axis of beam propagation. The images presented in this paper are realized using a scanner with 1024 dots on each A-Scan. The width of the scanning part of the skin was $12.8 \mathrm{~mm}$. During each horizontal pass over the skin, the piezoelectric element emits an impulse and receives the reflected 


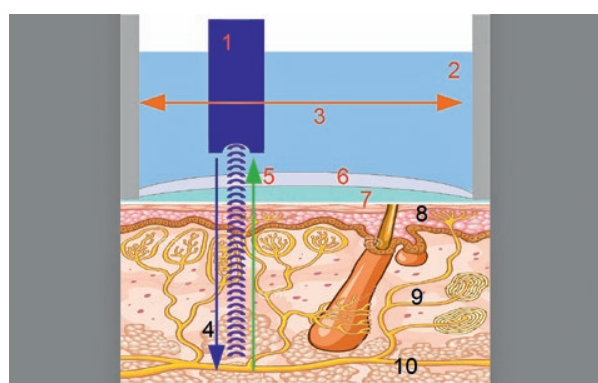

Fig 1. Diagram of the ultrasonic scanning of the skin: 1 - piezoelement, 2 - water in the applicator tip, 3 - direction of the ultrasound crystal movement, 4 - penetrating ultrasound wave, 5 - reflected ultrasound waves, 6 - membrane on the applicator tip, 7 - ultrasound contact gel, 8 - epidermis, 9 -dermis, 10 - subcutaneous fat.

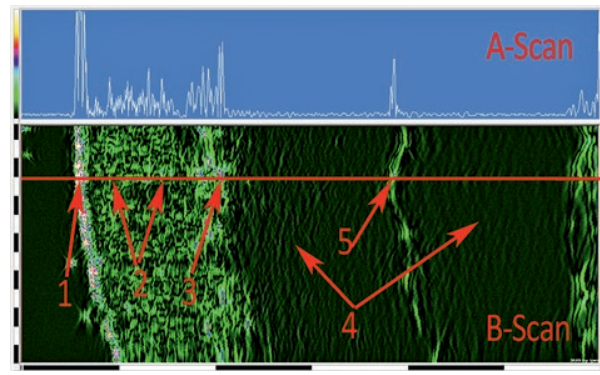

Fig 2. A-Scan and B-Scan: red horizontal line corresponds to the direction of penetration and the reflection of the ultrasonic beam. 1 - epidermis, 2 - dermis, 3 - lower dermis, border between dermis and subcutaneous fat, 4 - subcutaneous fat, 5 - superficial fascia.

signal 344 times, in steps of 33 microns. So, each B-Scan consisted of $344 \mathrm{~A}-\mathrm{Scans}$ reassembled in a two-dimensional image of $1024 \times 344$ pixels. Each A-Scan point of the tissue penetrated by a ultrasound beam, after digitizing is replaced by a color pixel. The intensity of luminescence or color of the pixel depends on the amplitude of the signal reflected from a given point. The color scale is shown to the left of A-scan (fig 2): low amplitude of the signal corresponds to the black and green color, average amplitude corresponds to blue and red, and high and maximum amplitude corresponds to orange, yellow, and white pixels. Inspected skin area has the length of 12.8 $\mathrm{mm}$ and a depth of 8-10 $\mathrm{mm}$ for $22 \mathrm{MHz}$ probe or 4-6 $\mathrm{mm}$ for $75 \mathrm{MHz}$ probe.

At the epidermis level there is an intense reflection of the ultrasound (maximum amplitude of the signal), in dermis there is a various degrees of reflected ultrasonic waves (heterogeneous signals) and at the fatty tissue level the reflection is much lower. Superficial fascia also has reflection and generally is visible in HFU.
Usually, all methods used for skin visualization are compared with the histological study of skin slices. HFU skin measurements have a strong correlation with histomorphometry due to its high resolution. The differences between the thickness of the epidermis and dermis obtained using histology and in vivo HFU come from the preparation of the tissue specimens with formaldehyde and dyes. The thicknesses of the skin layers in HFU may be considered more appropriate to the actual state of the tissue at the moment of study [3].

HFU permits the inspection of volumes of tissue at the microanatomical level anywhere on the body. Therefore, most experts agree that ultrasound is a method that builds a bridge between the subjective clinical inspection and palpation and histological micromorphological study.

In the present article we will describe some clinical cases with skin tumors evaluated by HFU. A digital ultrasound DUB SkinScanner (tpm GmbH, Germany) with 22 and $75 \mathrm{MHz}$ linear mechanical probes (axial resolution $72 \mu \mathrm{m}$ at $22 \mathrm{MHz}$ and $21 \mu \mathrm{m}$ at $75 \mathrm{MHz}$, lateral resolution $33 \mu \mathrm{m}$ for both probes, depth of scanning $10 \mathrm{~mm}$ for $22 \mathrm{MHz}$ and $5 \mathrm{~mm}$ for $75 \mathrm{MHz}$ ) was used. Vertical slicer cuts images of the skin $12.8 \mathrm{~mm} \times 10 \mathrm{~mm}$ for $22 \mathrm{MHz}$ and $12.8 \mathrm{~mm}$ x $5 \mathrm{~mm}$ for $75 \mathrm{MHz}$ were automatic reassembled for obtaining the two-dimensional imagines.

We drew schemes of histological slices of the skin for every HFU image in order to demonstrate the similarity between skin structure on HFU scans and micromorphological images. Due to this similarity in most cases it is possible to define the precise localization of lesions in the epidermis, dermis, and subcutaneous fat. This information has a high diagnostic value as it complements the clinical examination and shows the internal skin structure, the location of the tumor, and its boundaries with the surrounding tissue.

\section{Papillomas}

The depth of the rear contour of the papillomas is $300-600 \mu \mathrm{m}$, which corresponds to the papillary layer of the dermis. The tumor is clearly demarcated from the surrounding tissue and major part of the tumor usually stands out of the epidermis. Changes in the epidermis above the tumor are minimal. In some cases the contour of the epidermis could have a wavy shape, similar to the shape of the tumor. The thickness of the epidermis is unchanged compared with the normal epidermis. Most of the papillomas are hypoechoic - 0-3 units in comparison with the acoustic density of surrounding tissue- 16-24 units (fig 3).

Fibromas

Fibromas are located in the dermis, occupying the upper, medium, and deep layers of the dermis up to 2000- 


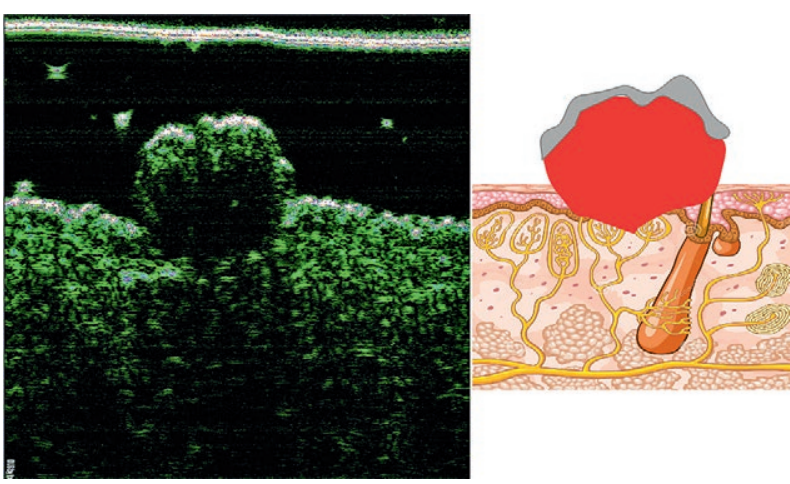

Fig 3. Scan of papilloma, $75 \mathrm{MHz}$ : scan on the left side, on the right side microanatomic scheme, demonstrating location of the tumor among the surrounding tissues. On the microanatomic scheme red color - area of the tumor, gray line - epidermis repeating contour of the tumor.

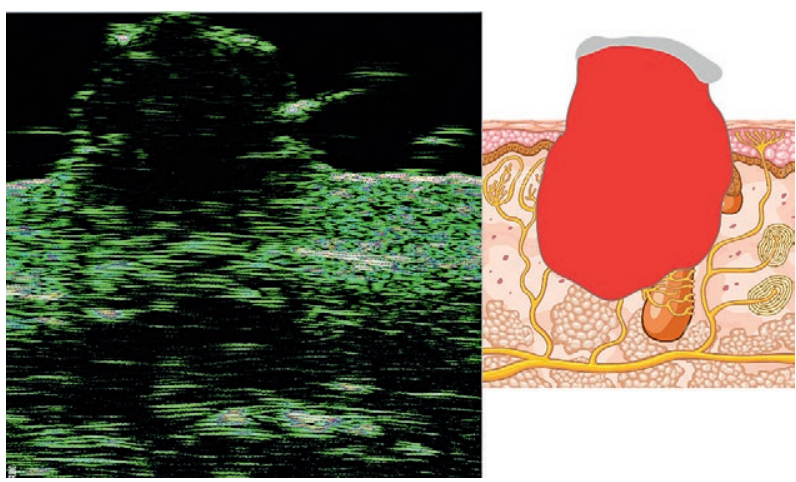

Fig 4. Scan of fibroma, $22 \mathrm{MHz}$ : scan on the left side, on the right side microanatomic scheme, demonstrating location of the tumor among the surrounding tissues. On the microanatomic scheme red color - area of the tumor, gray line - epidermis repeating contour of the tumor.

$2500 \mu \mathrm{m}$ from the skin surface. Delimitation of the tumor from surrounding tissues is clear. Around 1/3-1/4 of the tumor protrudes beyond the outer contour of the skin. The acoustic density of fibroma is reduced up to 0-3 units.. The epidermis of the new formation is not changed (fig 4).

\section{Keratofibromas}

The borders of the tumor can be detected and the rare contour of tumor corresponds to the upper layers of dermis and generally is $<500-600 \mu \mathrm{m}$. The acoustic density of keratofibroma is low (1-5 units). The epidermis of keratofibromas is thicker comparing with surrounding tissue (a mean of $230 \mu \mathrm{m}$ versus $110-105 \mu \mathrm{m}$ ). Acoustic density of the epidermis is increased up to 120 units (fig 5).

\section{Basaliomas}

In superficial basaliomas an increased thickness and acoustic density of the epidermis can be observed (180-

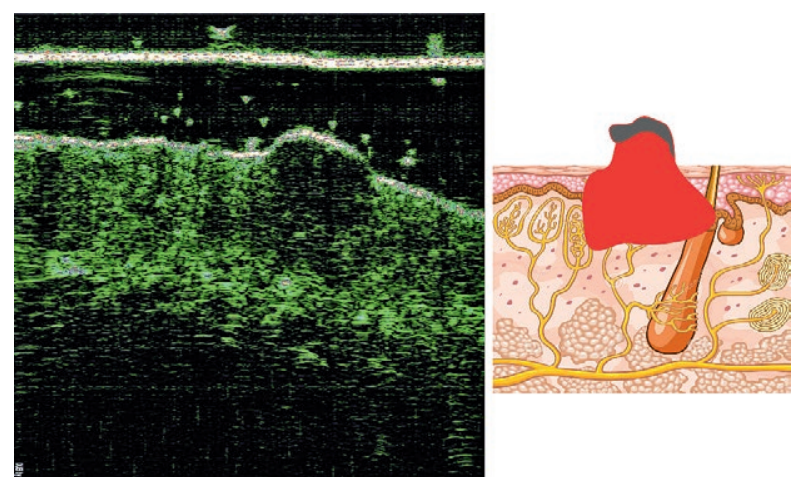

Fig 5. Scan of the keratofibroma, $75 \mathrm{MHz}$ : Scan on the left side, on the right side microanatomic scheme, demonstrating location of the tumor among the surrounding tissues. On the microanatomic scheme red color - area of the tumor, gray line - thickened epidermis.
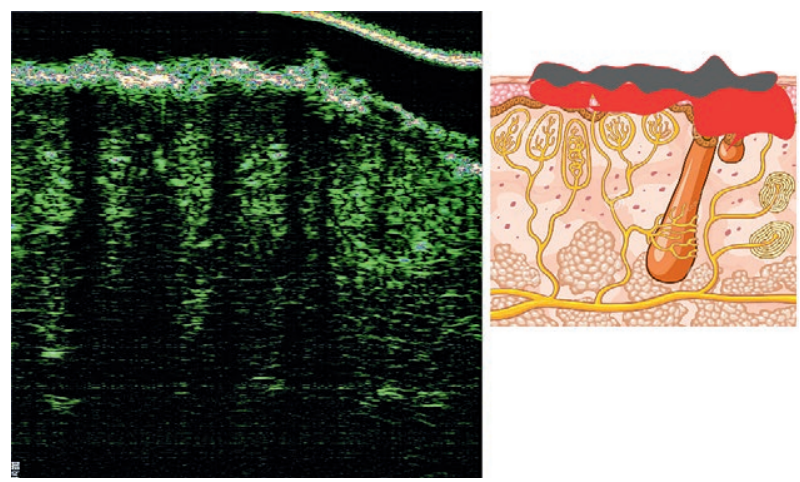

Fig 6. Scan of superficial BCC, $75 \mathrm{MHz}$ : scan on the left side, on the right side microanatomic scheme, demonstrating location of the tumor among the surrounding tissues. On the microanatomic scheme red color - hypoechoic subepidermal area, gray line - thickened epidermis.

$230 \mu \mathrm{m}$ and 120-130 units). In the subepidermal area a hypoechoic zone of 300-500 $\mu \mathrm{m}$ in depth can be detected (fig 6).

\section{Nodular basal cell carcinoma (BCC)}

Nodular BCC sometimes penetrates deep layers of the dermis. The tumor from figure 7 is visualized as an anechoic area (0-2 units acoustic density) with a depth of rear contour $2080 \mu \mathrm{m}$, width $10 \mathrm{~mm}$, and height $5 \mathrm{~mm}$. Epidermis is hyperechoic (120 units) and thickened up to 300 microns. For examination of the patients with $\mathrm{BCC}$ it is useful to combine the videodermoscopy with HFU. This combination succeeded in giving complementary and valuable information. With a videodermoscopy the pattern and the outer diameter of the tumor can be assessed, and HFU determines the depth of the rear contour of the tumor, its size, and boundaries within the intact tissue. 


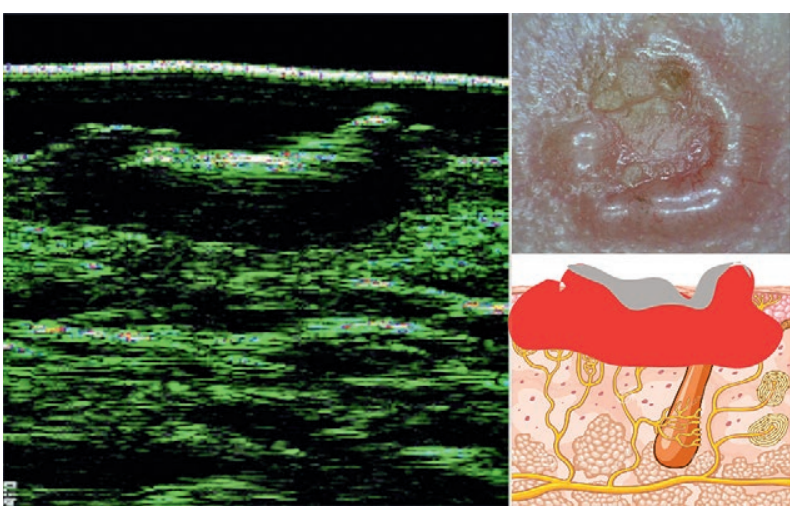

Fig 7. Scan of nodular BCC, $22 \mathrm{MHz}$ and videodermoscopy image. From the left: scan $22 \mathrm{MHz}$, videodermoscopy image and microanatomic scheme, demonstrating location of the tumor among the surrounding tissues. On the microanatomic scheme: red color - area of the tumor. The center of the tumor disintegrates, as determined on videodermatoscopy image, and the depth of necrosis and tumor borders are visible on scan.

\section{Discussions}

In HFU the skin tumors have a hypoechoic structure, even fibromas in which the connective tissue fibers provide unexpectedly low acoustic density. The acoustic density of dermis depends on water content, the quantity and balance of different collagen types, and on the spatial orientation of collagen and elastin fibers. The more expressed three-dimensional spatial orientation of collagen fibers in the dermis, the higher the echogenity $[4,5]$. The low acoustic density of the tumors $[6,7]$ could be explained by the loss of the three-dimensional fiber orientation and high homogeneity of the fibers and cells of the neoplasm.

One of the most valuable results of HFU is the precise definition of the rear contour of the tumor, the accurate measurement of of the tumor, especially within the skin, and the definition of boundaries within the unmodified tissue. This information can be obtained only with HFU, because by external examination, palpation, and dermoscopy these parameters cannot be clearly defined. Many researchers refer to HFU as the digital ultrasound biomicroscopy [8].

\section{Conclusion}

HFU study of the skin tumors allows the direct measurements of the size, depth, and boundaries of tumors. Data obtained are useful for carrying out the targeted biopsy and to choose the necessary intensity and depth of the impact for the treatment of skin tumors. Also, HFU is useful in monitoring the treatment response and the relapse of the tumor.

\section{Conflict of interest: none}

\section{References}

1. Schmid-Wendtner MH, Burgdorf W. Ultrasound scanning in dermatology. Arch Dermatol 2005; 141: 217-224.

2. Hoffmann K, Dirting K, Stücker M, el-Gammal S, Wilmert M, Altmeyer WP. Geschichte der hochfrequenten Sonographie. Ultraschall Med 1994; 15: 192-197.

3. De Rigal G, Escoffier C, Querleux B, Favire B, Agache P, Leveque JL. Assessment of aging of the human skin by in vivo ultrasonic imaging. J Investigative Dermatol 1989; 93: 621-625.

4. Gniadecka M, Gniadecki R, Serup J, Sondergaard J. U1trasound structure and digital image analysis of the subepidermal low echogenic band in aged human skin: diurnal changes and interindividual variability. J Invest Dermatol 1994; 102: 362-365.

5. Pohlhammer J, O'Brien WD Jr. Dependence of the ultrasonic scatter coefficient on collagen concentration in mammalian tissues. J Acoust Soc Med 1981; 69: 283-285.

6. Harland CC, Bamber JC, Gusterson BA, Mortimer PS. High frequency, high resolution B-scan ultrasound in the assessment of skin tumours. Br J Dermatol 1993; 128: 525-532.

7. Schmid-Wendtner MH, Burgdorf W. Ultrasound scanning in dermatology. Arch Dermatol 2005; 141: 217-224.

8. Foster FS, Pavlin CJ, Harasiewicz K, Christopher DA, Turnbull DH. Advances in ultrasound biomicroscopy. Ultrasound Med Biol 2000; 26: 1-27. 